\title{
ESTRESSE OCUPACIONAL E FATORES ASSOCIADOS ENTRE ENFERMEIROS DE HOSPITAIS PÚBLICOS*
}

\author{
Alessandro Rolim Scholze ${ }^{1}$, Julia Trevisan Martins², Maria Lucia do Carmo Cruz Robazzi ${ }^{3}$, Maria do Carmo \\ Fernandez Lourenço Haddad², Maria José Quina Galdino ${ }^{4}$, Renata Perfeito Ribeiro ${ }^{2}$
}

\begin{abstract}
RESUMO: Objetivou-se avaliar o estresse ocupacional e fatores associados entre enfermeiros de hospitais públicos. Estudo transversal com amostra de 185 enfermeiros de três hospitais públicos do Paraná. Os dados foram coletados entre novembro de 2015 e abril de 2016, mediante questionário de caracterização dos participantes e a Job Stress Scale. Realizaram-se análises descritivas e regressão logística. Entre os participantes, predominou o trabalho passivo e o de alta exigência. A percepção negativa dos serviços de apoio à assistência $(p=0,003)$, programas de educação continuada $(p=0,007)$, tempo e oportunidades para solucionar os problemas da assistência $(p<0,001)$ e participação em decisões administrativas $(p=0,014)$ aumentaram as chances de os enfermeiros perceberem o trabalho como estressante. Em contrapartida, maior tempo de trabalho na instituição $(p<0,001)$ e apoio social $(p<0,001)$ associaram-se a menores percepções de trabalho desgastante. Conclui-se que o estresse do enfermeiro esteve associado a fatores do ambiente laboral, sobretudo aqueles que dificultam prestar assistência de qualidade.
\end{abstract}

DESCRITORES: Saúde do trabalhador; Estresse psicológico; Enfermeiras e enfermeiros; Hospitais públicos.

\section{OCCUPATIONAL STRESS AND ASSOCIATED FACTORS AMONG NURSES AT PUBLIC HOSPITALS}

\begin{abstract}
We aimed to assess the occupational stress and associated factors among nurses from public hospitals. Cross-sectional study with a sample of 185 nurses from three public hospitals in Paraná. The data were collected between November 2015 and April 2016, using a questionnaire to characterize the participants and the Job Stress Scale. Descriptive analyses and logistic regression were applied. Among the participants, passive and high-demand work was predominant. The negative perception of the care support services $(p=0.003)$, continuing education programs $(p=0.007)$, time and opportunities to solve the care problems $(p<0.001)$ and participation in administrative $(p=0.014)$ increased the chances that the nurses would perceive the work as stressful. On the opposite, longer experience at the institution $(p<0.001)$ and social support $(p<0.001)$ were associated with lesser perceptions of exhausting work. In conclusion, the nurse's stress was associated with factors from the work environment, mainly factors that hamper highquality care delivery.
\end{abstract}

DESCRIPTORS: Occupational health; Stress, psychological; Nurses; Hospitals, public.

\section{ESTRÉS OCUPACIONAL Y FACTORES ASOCIADOS ENTRE ENFERMEROS DE HOSPITALES PÚBLICOS}

RESUMEN: = La finalidad fue evaluar el estrés ocupacional y factores asociados entre enfermeros de hospitales públicos. Estudio trasversal con muestra de 185 enfermeros de tres hospitales públicos de Paraná. Los datos fueron recolectados entre noviembre del 2015 y abril del 2016, mediante cuestionario de caracterización de los participantes y la Job Stress Scale. Fueron ejecutadas análisis descriptivas y regresión logística. Entre los participantes, predominó el trabajo pasivo y el de alta exigencia. La percepción negativa de los servicios de apoyo a la atención - $(p=0,003)$, programas de educación continuada $(p=0,007)$, tiempo y oportunidades para solucionar los problemas de la atención $(p<0,001)$ y participación en decisiones administrativas $(p=0,014)$ aumentaron las chances de que los enfermeros percibieran el trabajo como estresante. os enfermeiros perceberem o trabalho como estressante. Por otra parte, mayor tiempo de trabajo en la institución $(p<0,001)$ y apoyo social $(p<0,001)$ fueron asociados a menores percepciones de trabajo agotador. Se concluye que el estrés del enfermero estuvo asociado a factores del ambiente laboral, sobretodo aquellos que dificultan la prestación de atención de calidad.

DESCRIPTORES: Salud laboral; Estrés psicológico; Enfermeros; Hospitales públicos.

*Extraído da dissertação intitulada: "Ambiente de trabalho, consumo de substâncias psicoativas e estresse em enfermeiros hospitalares: análise dos fatores associados". Universidade Estadual de Londrina, 2016.

${ }^{1}$ Enfermeiro. Mestre em Enfermagem. Universidade Estadual de Londrina. Londrina, PR, Brasil

${ }^{2}$ Enfermeira. Doutora em Enfermagem. Docente de Enfermagem e do Programa de Pós-Graduação em Enfermagem da Universidade Estadual de Londrina. Londrina, PR, Brasil.

${ }^{3}$ Enfermeira. Doutora em Enfermagem. Docente de Enfermagem da Universidade de São Paulo. Ribeirão Preto, SP, Brasil.

${ }^{4}$ Enfermeira. Doutoranda em Enfermagem. Docente de Enfermagem da Universidade Estadual do Norte do Paraná. Bandeirantes, PR, Brasil. 
A enfermagem constitui-se na maior força de trabalho das instituições hospitalares e o trabalho desses profissionais é fundamental, pois são eles os responsáveis pelos cuidados diretos e ininterruptos ao paciente, durante as 24 horas do dia, sete dias por semana. O enfermeiro, na condição de gerente do cuidado, possui perspectiva única sobre o atendimento ao paciente e operações hospitalares, e está na linha de frente desses serviços de saúde ${ }^{(1-2)}$. No entanto, esses profissionais são submetidos a vários riscos ocupacionais, principalmente os psicossociais ${ }^{(3-4)}$.

Os enfermeiros vivem processo de trabalho árduo - físico e psicologicamente exaustivo - com demandas altas e complicadas e longas jornadas laborais; trabalham em turnos, com sobrecarga de trabalho motivada pelo insuficiente número de profissionais para atender às unidades superlotadas. Além disso, nesse ambiente, permeado de relações interpessoais complexas com a equipe multiprofissional, há falta de reconhecimento, autonomia e desvalorização da classe; há o convívio diário com o sofrimento, altas exigências de desempenho das equipes e para com a segurança do paciente. Esses e outros fatores desencadeiam o estresse ocupacional e produzem agravos à saúde física e mental desses profissionais ${ }^{(5-6)}$.

O estresse ocupacional é comumente interpretado como a sensação de estar tenso, sobrecarregado e preocupado em virtude das exigências laborais. Contudo, esse estresse é o resultado da interação entre demanda psicológica, controle/decisão sobre o trabalho ${ }^{(7)}$ e o apoio social que o trabalhador recebe em seu cotidiano laboral dos colegas e dos chefes. Nessa perspectiva teórica, o apoio social é entendido como o resultado da interação interpessoal no trabalho, que por proporcionar ambiente agradável, de apoio dos pares e das chefias de trabalho, produz efeito atenuante sobre as demandas psicológicas e a tensão laboral do trabalhador ${ }^{(8)}$.

Entre as principais repercussões associadas ao estresse ocupacional, têm-se o absenteísmo, insatisfação laboral, acidentes de trabalho, diminuição da qualidade de vida, síndrome de burnout, problemas cardiovasculares, distúrbios psíquicos menores, e também ocorre um declínio do desempenho do trabalhador, que impacta a qualidade do cuidado ${ }^{(9-13)}$.

Ante o exposto, torna-se importante que o enfermeiro e as organizações identifiquem os fatores estressantes do ambiente laboral, e também os prejuízos que a saúde física, mental e social desse profissional possa vir a sofrer. Essas informações poderão fornecer subsídios para se buscar soluções amenizadoras das situações de estresse do enfermeiro no ambiente laboral, e, assim, propiciar-lhe melhor qualidade de vida e bem-estar.

Diante dessas considerações, neste estudo objetivou-se avaliar o estresse ocupacional e os fatores associados entre enfermeiros de hospitais públicos.

\section{MÉTODO}

Estudo transversal, analítico-descritivo, desenvolvido em três hospitais públicos, aqui denominados A, B e C, localizados em um município do estado do Paraná. A instituição A é um hospital de nível terciário de atenção à saúde, com 313 leitos e 127 enfermeiros. Os hospitais B e C prestam assistência à saúde até o nível secundário, por meio de 130 e 113 leitos, respectivamente, e possuem 47 enfermeiros cada.

A população de estudo foi composta por 221 profissionais. Estabeleceu-se como critérios de inclusão: ser enfermeiro da assistência ou da supervisão; trabalhar em uma das instituições há pelo menos um ano, pois, após esse período, a maioria dos profissionais já se adaptou ao seu ambiente laboral; e não estar em licença do trabalho.

O cálculo amostral foi realizado por estratificação, considerando a proporção de $50 \%$, dado que a prevalência de estresse entre enfermeiros de hospitais públicos é desconhecida, o nível de confiança foi de $95 \%$ e o erro amostral absoluto de $5 \%$. Assim, o número mínimo de participantes nos hospitais secundários seria de 78, e no terciário, de 97 enfermeiros, perfazendo um total de 175 profissionais. 
todos os enfermeiros foram convidados a participar do estudo, destes, 13 (5,9\%) estavam em licença, $23(10,4 \%)$ se recusaram a participar e 185 (83,7\% -141 do período diurno e 44 do noturno) consentiram em participar, sendo 81 (36,7\%) dos hospitais secundários e 104 (47,0\%) do hospital terciário.

A coleta de dados foi realizada entre novembro de 2015 e abril de 2016, e os profissionais foram abordados pelo pesquisador principal durante os intervalos dos plantões, convidando-os a participar da pesquisa. Aqueles que demonstravam interesse eram direcionados para um ambiente reservado e esclarecidos sobre o estudo, e após o consentimento lhes foi entregue o instrumento de coleta de dados em envelope individual. Ao término do preenchimento do instrumento, o participante depositava os envelopes em urna lacrada, a fim de garantir confidencialidade.

O desenvolvimento do estudo ocorreu em conformidade às normas vigentes de ética em pesquisa envolvendo seres humanos, sendo aprovado pelo Comitê de Ética em Pesquisa da Universidade Estadual de Londrina, sob o Parecer n. 1.310.741 e CAAE: 49062415.5.0000.5231.

O instrumento de coleta de dados foi composto por dois questionários: o de caracterização dos participantes e a versão brasileira da Job Stress Scale, traduzida e validada em $2004^{(14)}$.

O primeiro instrumento continha questões de caracterização sociodemográfica (sexo; idade; renda; estado civil; filhos; e titulação), ocupacional (tempo de trabalho na instituição; jornada laboral semanal; cargo e turno de atuação; outros vínculos laborais; percepção sobre: 1. serviços de apoio à assistência, 2. eficácia da educação continuada, 3. tempo e oportunidades para discutir com outros colegas os cuidados prestados ao paciente e a resolução de problemas relacionados à assistência, e 4 . participação nas decisões administrativas), e de hábitos de vida (prática de atividade física, consumo de álcool e tabaco). Esse questionário foi elaborado pelos autores e testado previamente em uma amostra de 20 enfermeiros não integrantes da população do estudo, a fim de verificar sua compreensão e operacionalidade.

A Job Stress Scale é uma escala autoaplicável, constituídade 17 questões, com respostas do tipo Likert, que avalia o estresse ocupacional por meio de três dimensões: demandas psicológicas (cinco itens), controle no processo de trabalho (seis itens) e apoio social (seis itens). A dimensão apoio social integra a escala, mas tem a hipótese de amortecer a associação entre a tensão do trabalho e distúrbios relacionados ao estresse, além disso, constitui-se como uma variável (dimensão) latente ${ }^{(8,14)}$.

A partir da combinação das dimensões demanda e controle, há uma indicação do tipo de trabalho que o profissional experencia: (1) trabalho de alta exigência, considerado estressante (baixo controle e alta demanda); (2) trabalho passivo (baixo controle e baixa demanda); (3) trabalho ativo (alto controle e alta demanda); e (4) trabalho de baixa exigência (alto controle e baixa demanda). O trabalho de baixa exigência e o ativo constituem-se em experiências laborais ideais ao trabalhador, pois são motivadores e favorecem a criatividade. Por outro lado, o trabalho de alta exigência e o passivo são considerados os mais prejudiciais, pois há maior exigência psíquica, predispondo o trabalhador a desenvolver comorbidades, conforme se esquematiza na Figura $1^{(15)}$.

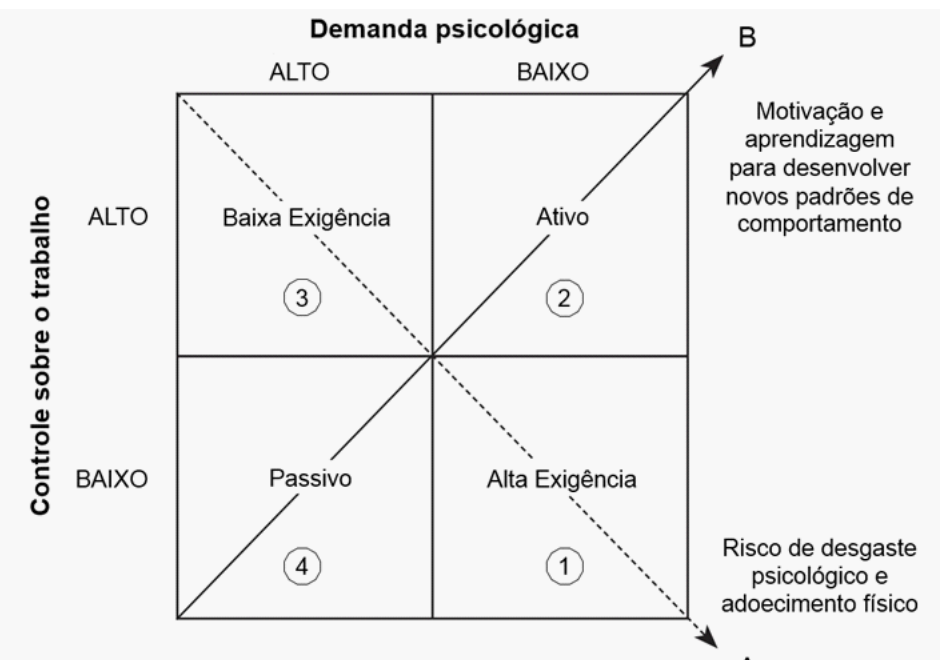

A

Figura 1 - Modelo de relação entre controle e demanda, adaptado de Theorell; Karasek. Washington, DC, Estados Unidos, 1996 
Os dados foram analisados no programa Statistical Package of Social Sciences ${ }^{T M}$ (SPSS), versão 20.0. Foram realizadas análises descritivas, de associação bivariada e de regressão logística bruta e ajustada, com cálculo de odds ratio (OR) e respectivos intervalos de confiança (IC 95\%). Para o modelo multivariado, por meio do método backward, foram incorporadas todas as variáveis independentes que apresentaram $\mathrm{p} \leq 0,20$ na análise bivariada, considerando-se o trabalho de alta exigência como variável dependente. Permaneceram no modelo as variáveis independentes que mantiveram associação significativa após o ajuste $(\mathrm{p}<0,05)$, de acordo com o teste de Wald.

\section{- RESULTADOS}

Dos 185 enfermeiros que participaram deste estudo, a maioria pertencia ao sexo feminino ( $n=152 ; 82,2 \%)$, possuía relacionamento conjugal estável ( $n=113 ; 61,1 \%)$ e filho(s) $(n=115 ; 62,2 \%)$. A idade variou entre 24 e 63 anos, com média de 41,25 anos, e a renda mensal individual média foi de R\$6.596,42, variando entre $\mathrm{R} \$ 2.300,00$ e $\mathrm{R} \$ 17.600,00$.

Em relação à caracterização ocupacional, prevaleceram enfermeiros com apenas um vínculo empregatício ( $n=137 ; 74,1 \%)$, que atuavam em cargos assistenciais ( $n=136 ; 73,5 \%)$ e no período diurno $(n=141 ; 76,2 \%)$. O tempo de trabalho na instituição variou entre um e 34 anos, com média de 7,99. A carga horária semanal de trabalho dos enfermeiros com vínculo único foi de 37,8 horas, em média, com variação de 24 a 44 horas. Entre aqueles com mais de um vínculo, a média foi de 69,4 horas, e variou entre 50 e 84 horas.

Sobre os hábitos de vida, identificou-se que a maioria dos participantes praticavam atividade física $(n=101 ; 54,6 \%)$, e a minoria consumia bebidas alcoólicas com frequência $(n=38 ; 20,5 \%)$, e era tabagista $(\mathrm{n}=32 ; 17,3 \%)$.

Na Figura 2 apresenta-se o tipo de trabalho realizado pelos participantes, conforme a categorização do modelo de Demanda-Controle, constatando-se o predomínio de enfermeiros que desenvolvem um trabalho passivo e de alta exigência.

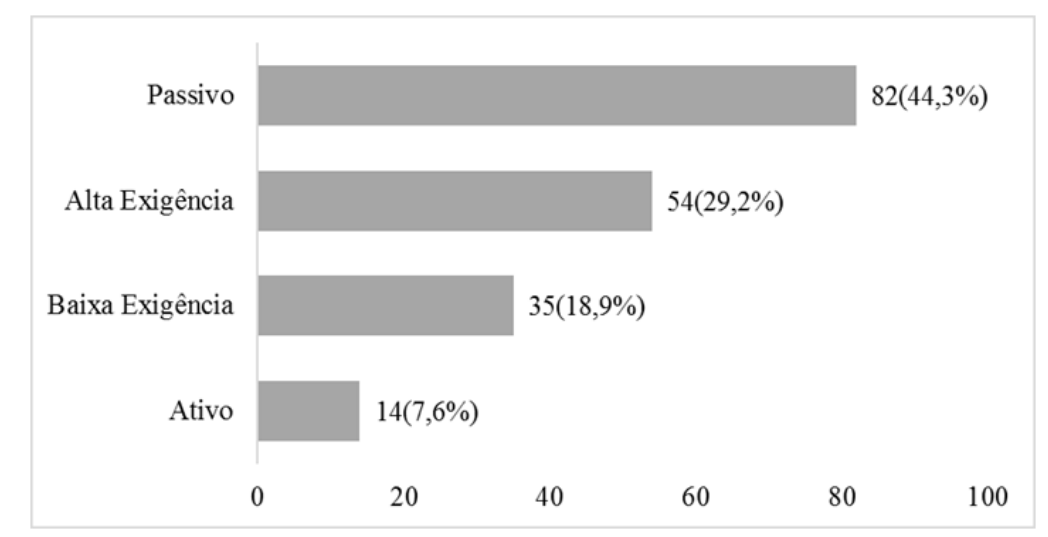

Figura 2 - Distribuição dos tipos de trabalho percebidos pelos enfermeiros hospitalares, de acordo com o modelo Demanda-Controle ( $\mathrm{n}=185$ ). Londrina, PR, Brasil, 2015-2016

Considerando-se que o trabalho de alta exigência (baixo controle e alta demanda) é aquele que representaalto risco deadoecimento físico e mental do trabalhador, foi ajustado um modelo multivariado $(p<0.001)$ que identificou os fatores associados (Tabela 1). A percepção negativa sobre os serviços de apoio à assistência $(\mathrm{OR}=3,78)$, eficácia dos programas de educação continuada (OR=3,78), tempo e oportunidades para discutir com outros colegas os problemas oriundos dos cuidados prestados ao paciente $(O R=4,41)$ aumentaram significativamente as chances de os enfermeiros perceberem seus trabalhos como estressantes.

Por outro lado, enfermeiros que revelaram percepção negativa quanto à participação em decisões administrativas $(\mathrm{OR}=0,28)$ apresentaram menor chance de perceberem seu trabalho como de alta 
exigência, isto é, a não participação em decisões administrativas reduziu a percepção de trabalho estressante.

Também se constatou que, quanto maior o tempo de trabalho na instituição $(O R=0,89)$ e a percepção de apoio social recebida pelos chefes e colegas no trabalho $(\mathrm{OR}=0,75)$, menores foram as percepções de trabalho desgastante.

Tabela 1 - Fatores associados ao trabalho de alta exigência entre enfermeiros de instituições hospitalares públicas $(\mathrm{n}=185)$. Londrina, PR, Brasil, 2015-2016

\begin{tabular}{|c|c|c|c|}
\hline \multirow[t]{2}{*}{ Variáveis } & \multicolumn{3}{|c|}{ Trabalho de alta exigência } \\
\hline & $\mathbf{n}(\%)$ & OR ajustado (IC 95\%)* & p-value \\
\hline \multicolumn{4}{|c|}{ Serviços de apoio à assistência } \\
\hline Percepção negativa & $41(46,1)$ & $3,78(1,56-9,18)$ & 0,003 \\
\hline Percepção positiva & $13(13,5)$ & 1 & \\
\hline \multicolumn{4}{|c|}{ Eficácia da educação continuada } \\
\hline Percepção negativa & $19(35,2)$ & $3,18(1,36-7,45)$ & 0,007 \\
\hline Percepção positiva & $35(64,8)$ & 1 & \\
\hline \multicolumn{4}{|c|}{ Participação nas decisões administrativas } \\
\hline Percepção negativa & $30(55,6)$ & $0,28(0,10-0,77)$ & 0,014 \\
\hline Percepção positiva & $24(44,4)$ & 1 & \\
\hline \multicolumn{4}{|c|}{ Tempo e oportunidades para discutir com outros colegas os cuidados prestados ao paciente } \\
\hline Percepção negativa & $44(81,5)$ & $4,41(1,62-12,01)$ & $<0,001$ \\
\hline Percepção positiva & $10(18,5)$ & 1 & \\
\hline Tempo de trabalho & $7,48(8,06)^{* *}$ & $0,89(0,83-0,96)$ & $<0,001$ \\
\hline Apoio social & $17,9(2,9)^{* *}$ & $0,75(0,63-0,90)$ & $<0,001$ \\
\hline
\end{tabular}

*odds ratio ajustado (intervalo de confiança 95\%); **Média (desvio padrão)

\section{- DISCUSSÃO}

No presente estudo houve predominância de mulheres, jovens, com relacionamento conjugal estável e com filhos. Denota-se que essas mulheres estão expostas à dupla jornada de trabalho, considerandose que realizam as atividades inerentes ao ambiente de trabalho e às atribuições domésticas - cuidar do lar e dos filhos ${ }^{(16)}$. Dessa forma, deve-se considerar o desgaste devido à sobrecarga de atribuições, tanto no local de trabalho quanto no seu cotidiano familiar, o que faz aumentar a suscetibilidade ao estresse.

Em relação aos hábitos de vida dos participantes deste estudo, constatou-se que a maioria praticava atividade física e uma minoria consumia bebidas alcoólicas com frequência e era tabagista. A adoção desse perfil salutar pode estar associada ao fato de esses profissionais conhecerem os efeitos prejudiciais do sedentarismo e das substâncias psicoativas nos níveis biopsicossociais, que incluem: obesidade, diabetes mellitus, doenças cardiovasculares, depressão, distúrbios do sono, dependência de substâncias, entre outras ${ }^{(17)}$.

A maioria dos enfermeiros deste estudo possuía apenas um vínculo de trabalho ( $n=137 ; 74,1 \%$ ) e carga horária semanal média de 37,84 horas, provavelmente por apresentarem uma média salarial razoável e estabilidade no emprego por serem concursados. Essa condição é considerada próxima da ideal, pois, por não desenvolveram longas jornadas laborais, mantêm os níveis de alerta, diminuindo os riscos de eventos adversos, acidentes de trabalho, absenteísmo e presenteísmo, exaustão laboral e doenças de natureza mental que, por sua vez, são causas importantes de incapacidade permanente ${ }^{(17-18)}$. Entretanto, tais aspectos negativos podem ocorrer com os 48(25,9\%) enfermeiros desta amostra que 
indicaram duplo vínculo e que desenvolviam uma jornada semanal de trabalho média de 69,4 horas, com variação de 50 a 84 horas.

Embora a maioria desenvolva suas atividades laborais no turno diurno, deve-se ponderar que $23,8 \%(n=44)$ trabalhavam no período noturno, inevitável na área hospitalar, pois o cuidado ao paciente deve ser contínuo. Esse tipo de trabalho provoca alterações fisiológicas e afeta o bem-estar físico e psicológico dos profissionais, predispondo o trabalhador à fadiga, diminuição do desempenho e insatisfação laboral, levando-os ao desgaste e à doença ${ }^{(10,19-20)}$. Neste estudo o trabalho noturno não compôs o modelo múltiplo, por não apresentar associação significativa com o estresse ocupacional, o que também foi observado por estudo com enfermeiros taiwaneses. Contudo, identificou-se que o trabalho em turnos, principalmente realizado à noite, está relacionado à pior qualidade do sono ${ }^{(21)}$.

O estresse é desencadeado no trabalhador quando ele não consegue manter o controle sobre sua prática, podendo apresentar um trabalho de alta exigência ou passivo, que converge para o adoecimento ou para o distanciamento de suas atividades laborais, respectivamente ${ }^{(14)}$.

Entre os enfermeiros deste estudo, constatou-se predominantemente o trabalho passivo, seguido daquele de alta exigência, dados que se assemelham aos de outras investigações realizadas no Brasil e exterior ${ }^{(11,16)}$. Infere-se que tais condições podem levar os trabalhadores à redução gradual na capacidade de solucionar problemas rotineiros em seu ambiente laboral, além de vivenciar níveis altos de sofrimento e de insatisfação devido à monotonia do processo de trabalho, que desapropria o trabalhador do seu know-how ${ }^{(22)}$, tornando-o incapaz de enfrentar os desafios intelectuais que podem surgir no cotidiano do trabalho em enfermagem.

O trabalho de alta exigência, caracterizado por baixo controle e alta demanda, é o que predispõe o trabalhador ao adoecimento físico e mental. Entre as variáveis preditoras que compuseram o modelo multivariado, todas foram de gênese ocupacional, sobretudo as relacionadas à assistência prestada aos pacientes.

Os enfermeiros deste estudo indicaram que a insatisfação com os serviços de apoio à assistência $(\mathrm{OR}=3,78 ; \mathrm{p}=0,003)$, com os programas de educação continuada $(\mathrm{OR}=3,18 ; \mathrm{p}=0,007)$ e o tempo e oportunidades insuficientes para discutir com outros colegas os cuidados prestados ao paciente $(\mathrm{OR}=4,41 ; \mathrm{p}<0,001)$ foram fatores que aumentaram as chances de manisfestação do estresse ocupacional. Ressalta-se que as atividades desenvolvidas na área hospitalar são complexas e dinâmicas, exigindo do profissional domínio de tecnologias, conhecimento e habilidades específicas. Entretanto, esse ambiente também é permeado pela precarização das condições de trabalho e do sistema de saúde, evidenciado na escassez de recursos humanos e materiais, na superlotação das unidades e na fragmentação do processo de trabalho ${ }^{(23)}$.

Esse "modus operandi" repercute na qualidade da assistência prestada pelo enfermeiro, pois, pela quantidade insuficiente de trabalhadores frente à alta demanda de pacientes, não há tempo hábil para desenvolver assistência adequada ao paciente e ao familiar. Em consequência, têm-se complicações do quadro clínico do paciente, eventos adversos, aumento nas taxas de infecção, lesões por pressão, quedas, aumento do período de internação e dos custos, ou seja, prezuízos aos pacientes, às instituições de saúde e aos profissionais ${ }^{(13,24)}$.

Embora haja avanços na compreensão de que o cuidado é interdisciplinar, a tomada de decisão sobre metas do cuidado com os pacientes e suas famílias é, histórica e culturalmente, atribuída ao médico. Assim, é comum nos serviços de saúde o enfermeiro não ser apoiado para se envolver na comunicação e na tomada de decisões sobre os objetivos e metas do cuidado. Entretanto, esse profissional tem se empoderado da sua prática laboral, vislumbrado melhorar a qualidade dos cuidados de saúde e, portanto, ele deve se inserir na tomada de decisão compartilhada, pelo fato de compor a equipe multidisciplinar de saúde e também porque a enfermagem é uma ciência que envolve competências e habilidades específicas ${ }^{(25)}$.

Nesse sentido, compreende-se a importância da educação continuada nas instituições hospitalares como ferramenta para capacitar, desenvolver e aprimorar a competência técnico-científica do profissional, a qual, ao aumentar a segurança e habilidade para a prática diária ${ }^{(26)}$, amenizará a percepção do trabalho estressante. 
Essa assertiva foi corroborada por investigação realizada na Islândia, que avaliou o impacto de um programa educacional de capacitação em enfermagem sobre a percepção da demanda-controleapoio social de enfermeiros hospitalares. Essa investigação identificou que, entre os enfermeiros que caracterizaram o trabalho como de alta exigência, o programa educacional de capacitação contribuiu para que eles o considerassem menos estressante, pois, ao aumentar a autonomia e o controle dos enfermeiros sobre seu processo de trabalho, a capacitação também aumentou o apoio social recebido de seus administradores e colegas de trabalho ${ }^{(27)}$.

Citam-se, além dos métodos tradicionais de educação continuada no serviço, as opções: treinamentos baseados em simulação realística; cursos de capacitação on-line; e ajudas de decisão rápida presentes em sítios da web e aplicativos ${ }^{(25)}$. Ademais, com o advento da enfermagem de prática avançada, os mestrados profissionais surgem como alternativa relevante de capacitação, à medida que formam profissionais para uma prática transformadora, inovadora e avançada de procedimentos e processos, com a incorporação de evidências científicas ${ }^{(28)}$.

Salienta-se que o desenvolvimento de ações de educação continuada nas instituições hospitalares é de responsabilidade dos gestores e, desse modo, qualquer atividade educativa que resulte no aperfeiçoamento profissional deve ser incentivada e apoiada, independente se realizada dentro ou fora do ambiente de trabalho.

Os enfermeiros que participavam de decisões administrativas apresentaram maior chance de perceberem seu trabalho como de alta exigência quando comparados aos que não participavam $(\mathrm{OR}=0,28 ; p=0,014)$, o que também foi constatado em estudo com enfermeiros suecos. Ainda que os enfermeiros gerentes possuam maior controle sobre seu trabalho, o que pode camuflar o estresse, apresentam carga de trabalho maior, ritmo laboral mais acelerado e níveis elevados de exigências psicológicas, aumentando sua vulnerabilidade ao adoecimento quando comparados aos enfermeiros assistenciais $^{(2)}$.

No presente estudo, os enfermeiros com maior tempo de trabalho na instituição apresentaram menores chances de trabalho desgastante $(O R=0,89 ; p<0,001)$. Apesar de o envelhecimento do trabalhador diminuir sua capacidade física e cognitiva, os anos de carreira lhe conferem maior experiência profissional e resiliência para lidar com o estresse ${ }^{(12)}$. Nesse sentido, torna-se importante reconhecer esses profissionais na instituição, e incentivá-los a compartilhar sua experiência com os pares, auxiliando-os a enfrentar e a desenvolver estratégias de defesa frente ao estresse ocupacional.

Também se constatou, neste estudo, que as menores percepções de apoio social recebido dos chefes e colegas no trabalho associaram-se ao trabalho estressante $(O R=0,75 ; p<0,001)$. Outros estudos realizados demonstraram que o clima organizacional favorável - especialmente o apoio instrumental e as relações emocionais estabelecidas com os chefes e colegas - produz efeitos atenuantes sobre o estresse que ajudam o trabalhador no enfrentamento de situações difíceis, tensas e problemáticas ${ }^{(8-9,15)}$. A cultura organizacional, falta de apoio ao trabalhador, falha na comunicação e conflitos entre médicos, enfermeiros e outros profissionais são fatores que contribuem para o adoecimento mental e o desgaste nas relações interpessoais no contexto hospitalar ${ }^{(6)}$.

Diante das repercussões negativas do estresse no enfermeiro, são necessárias intervenções nos processos organizacionais para reduzir estressores específicos do ambiente hospitalar: mudanças nas condições de trabalho, na organização do apoio à assistência, no aumento das habilidades de comunicação e na mudança dos horários de trabalho ${ }^{(29)}$. Também se deve ponderar sobre as técnicas de relaxamento físico e mental, considerando-as estratégias individuais para a redução do estresse ocupacional, apesar de serem menos efetivas do que as organizacionais ${ }^{(30)}$.

Os achados desta pesquisa sugerem a necessidade de as instituições hospitalares investirem nos serviços de apoio à assistência, nos programas de educação continuada, na disponibilidade de espaços coletivos multidisciplinares para solucionar os problemas do cuidado ao paciente, e promoverem o apoio social no ambiente laboral dos enfermeiros hospitalares, visando diminuir-lhes o estresse.

As limitações do estudo são intrínsecas ao recorte transversal, que não possibilita estabelecer relações de causalidade. Entretanto, acredita-se que este estudo traz contribuições relevantes, pois, ao identificar os fatores que predizem o estresse prejudicial ao enfermeiro, permite que os gestores e os próprios trabalhadores busquem medidas para amenizá-lo e, assim, melhorar a qualidade de vida no 
trabalho e, por sua vez, a assistência prestada aos pacientes e familiares.

Os achados desta investigação não são generalizáveis. Portanto, sugere-se a realização de estudos em outras regiões do país, sobretudo entre enfermeiros de instituições privadas, pelas peculiaridades do seu processo de trabalho.

\section{- CONCLUSÃO}

Houve predominância do trabalho passivo e de alta exigência na amostra investigada, indicando consequências negativas para a saúde física e mental do enfermeiro. Os fatores associados ao trabalho de alta exigência foram a percepção negativa em relação aos serviços de apoio à assistência, aos programas de educação continuada, ao pouco tempo e às oportunidades para solucionar os problemas advindos do cuidado e à participação em decisões administrativas. O maior tempo de trabalho na instituição e a percepção de apoio social relacionaram-se às menores percepções de trabalho desgastante.

\section{AGRADECIMENTOS}

À Coordenação de Aperfeiçoamento de Pessoal de Nível Superior (CAPES) e Fundação Araucária pelo apoio financeiro a esta pesquisa.

\section{REFERÊNCIAS}

1. Dagget T,Molla A,Belachew T. Job related stress among nurses working in Jimma Zone public hospitals, South West Ethiopia: a cross sectional study. BMC Nurs.[Internet] 2016;(15)[acesso em 02 abr 2017]. Disponível: http:// dx.doi.org/10.1186/s12912-016-0158-2.

2. Gunilla J,Sandahl C,Hasson D. Role stress among first-line nurse managers and registered nurses - a comparative study. J Nurs Manag.[Internet] 2013; 21(3) [acesso em 02 abr 2017].Disponível: http://dx.doi.org/10.1111/j.13652834.2011.01311.x.

3. Chiou ST,Chiang JH,Huang N,Wu CH,Chien LY. Health issues among nurses in Taiwanese hospitals: National survey.Int J Nurs Stud. [Internet] 2013;50(10)[acesso em 02 abr 2017]. Disponível: http://dx.doi.org/10.1016/j. ijnurstu.2013.01.012.

4. Tung CY,Chang CC,Ming JL,Chao KP. Occupational hazards education for nursing staff through web-based learning. Int. J. Environ. Res. Public Health.[Internet] 2014;11(12) [acesso em 02 abr 2017]. Disponível: http:// dx.doi.org/10.3390/ijerph111213035.

5. Sarafis P, Rousaki E, Tsounis A, Malliarou M, Lahana L, Bamidis $P$, et al. The impact of occupational stress on nurses' caring behaviors and their health related quality of life. BMC Nurs. [Internet] 2016;(15) [acesso em $02 \mathrm{abr}$ 2017]. Disponível: http://dx.doi.org/10.1186/s12912-016-0178-y.

6. Adib-Hajbaghery M,Khamechian M,Alavi NM. Nurses' perception of occupational stress and its influencing factors: a qualitative study. Iran J Nurs Midwifery Res. [Internet]2012;17(5) [acesso em 02 abr 2017]. Disponível: https://www.ncbi.nlm.nih.gov/pmc/articles/PMC3703075/.

7. Karasek RA. Job Demand, job decision latitude, and mental strain: implications for job redesign. Admin Sci Quar. [Internet] 1979;24(2) [acesso em 01 jun 2017]. Disponível: http://dx.doi.org/10.2307/2392498.

8. Johnson JV, Hall EM. Job strain, workplace social support, and cardiovascular disease: a cross-sectional study of a random sample of the Swedish working population. Am J Public Health. [Internet] 1988;78(10)[acesso em 12 jun 2017]. Disponível: http://dx.doi.org/10.2105/AJPH.78.10.1336.

9. Urbanetto JS, Magalhaes MCC, Maciel VO, Sant'anna VM, Gustavo AS, Poli-de-Figueiredo CE, et al. Workrelated stress according to the demand-control model and minor psychic disorders in nursing workers. Rev. esc. enferm. USP. [Internet] 2013;47(5) [acesso em 02 abr 2017]. Disponível: http://dx.doi.org/10.1590/S0080623420130000500024. 
10. Ferri P,Guadi M,Marcheselli L,Balduzzi S,Magnani D,Di Lorenzo R. The impact of shift work on the psychological and physical health of nurses in a general hospital: a comparison between rotating night shifts and day shifts. Risk Manag Health Policy.[Internet] 2016;(9)[acesso em 02 abr 2017]. Disponível: http://dx. doi. org/10.2147/RMHP.S115326.

11. Pisanti R,Van Der Doef M,Maes S, Meier LL, Lazzari D, Violani C. How changes in psychosocial job characteristics impact burnout in nurses: a longitudinal analysis. Front. Psychol.[Internet] 2016;7(1082) [acesso em 02 abr 2017].Disponível: http://dx.doi.org/10.3389/fpsyg.2016.01082.

12. Yang T,Shen YM,Zhu M,Liu Y,Deng J,Chen Q,et al. Effects of co-worker and supervisor support on job stress and presenteeism in an aging workforce: a structural equation modelling approach. Int J Environ Res Public Health.[Internet] 2016;13(1) [acesso em 02 abr 2017]. Disponível: http://dx.doi.org/10.3390/ijerph13010072.

13. Hessels AJ,Flynn L, Cimiotti JP,Cadmus E,Gershon RRM. The impact of the nursing practice environment on missed nursing care. Clin Nurs Stud.[Internet] 2015;3(4) [acesso em 02 abr 2017]. Disponível: http://dx.doi. org/10.5430/cns.v3n4p60.

14. Alves MGM, Chor D, Faerstein E, Lopes CS, Werneck GL. Short version of the "job stress scale": a Portugueselanguage adaptation. Rev. Saúde Pública. [Internet] 2004;38(2) [acesso em 02 abr 2017]. Disponível: http://dx.doi. org/10.1590/S0034-89102004000200003.

15.Theorell T, Karasek RA. Current issues relating to psychosocial job strain and cardiovascular diseasse research. J Occup Health Psychol. [Internet]1996;1(1) [acesso em 02 abr 2017]. Disponível: http://dx.doi.org/10.1037/10768998.1.1.9.

16. Prochnow A, Magnago TSBS, Urbanetto JS, Beck CLC, de Lima SBS, Greco PBT. Work ability in nursing: relationship with psychological demands and control over the work. Rev. Latino-Am. Enfermagem. [Internet] 2013;21(6) [acesso em 02 abr 2017]. Disponível: http://dx.doi.org/10.1590/0104-1169.3072.2367.

17. Chin DL, Nam S, Lee SJ. Occupational factors associated with obesity and leisure-time physical activity among nurses: A cross sectional study. Int J Nurs Stud. [Internet] 2016;(57)[acesso em 02 abr 2017]. Disponível: http:// dx.doi.org/10.1016/j.ijnurstu.2016.01.009.

18. Hannerz H, Albertsen K. Long working hours and subsequent use of psychotropic medicine: a study protocol. JMIR Res Protoc.[Internet] 2014;3(3) [acesso em 02 abr 2017]. Disponível: http://dx.doi.org/10.2196/resprot.3301.

19. Gómez-GarcíaT, Ruzafa-Martínez M, Fuentelsaz-Gallego C, Madrid JA, Rol MA,Martínez-Madrid MJ, et al. Nurses' sleep quality, work environment and quality of care in the Spanish National Health System: observational study among different shifts. BMJ Open. [Internet] 2016;6(8)[acesso em 02 abr 2017]. Disponível: http://dx.doi. org/10.1136/bmjopen-2016-012073.

20. Silva SM, Baptista PCP. Novosolharessobre o sujeito que adoece no trabalhohospitalar. Cogitare Enferm. [Internet] 2013;18(1) [acesso em 02 abr 2017]. Disponível: http://dx.doi.org/10.5380/ce.v18i1.25332.

21. Lin SH,Liao WC, Chen MY,Fan JY. The impact of shift work on nurses' job stress, sleep quality and selfperceived health status. J Nurs Manag.[Internet] 2014;22(5) [acesso em 01 jun 2017]. Disponível: http://dx.doi. org/10.1111/jonm.12020.

22. Dejours C, Abdoucheli E, Jayet C. Psicodinâmica do trabalho: contribuição da escola dejouriana à análise da relação prazer, sofrimento e trabalho. São Paulo: Atlas; 2014.

23. Maciel RHMO, dos Santos JBF, Rodrigues RL. Healthcare workers' work conditions: a perspective on technicians and midlevel workers.Rev. bras. saúde ocup. [Internet] 2015;40(131) [acesso em 02 abr 2017]. Disponível: http:// dx.doi.org/10.1590/0303-7657000078613.

24. Yang $\mathrm{PH}, \mathrm{Hung} \mathrm{CH}, \mathrm{Chen} \mathrm{YM}, \mathrm{Hu} \mathrm{CY}$, Shieh SL. The impact of different nursing skill mix models on patient outcomes in a respiratory care center. Worldviews Evid Based Nurs.[Internet] 2012;9(4) [acesso em 02 abr 2017 ]. Disponível: http://dx.doi.org/10.1111/j.1741-6787.2012.00246.x.

25. You JJ, Downar J, Fowler RA, Lamontagne F,Ma IW,Jayaraman D, et al. Barriers to goals of care discussions with seriously ill hospitalized patients and their families: a multicenter survey of clinicians. JAMA Intern Med. [Internet] 2015;175(4) [acesso em 02 abr 2017]. Disponível: http://dx.doi.org/10.1001/jamainternmed.2014.7732. 
26. Shahhosseini Z,Hamzehgardeshi Z. The facilitators and barriers to nurses' participation in continuing education programs: a mixed method explanatory sequential study. Glob J Health Sci.[Internet] 2015;7(3) [acesso em 02 abr 2017]. Disponível: http://dx.doi.org/10.5539/gjhs.v7n3p184.

27. Sigurdardottir AO,Svavarsdottir EK,Juliusdottir S. Family nursing hospital training and the outcome on job demands, control and support. Nurse Educ Today.[Internet] 2015;35(7) [acesso em 02 abr 2017]. Disponível: http:// dx.doi.org/10.1016/j.nedt.2015.03.003.

28. Scochi CGS, Gelbcke FL, Ferreira MA, Alvarez AM. Professional Master's Degree: potential contribution to Advanced Practice Nursing. Rev Bras Enferm. [Internet] 2015;68(6) [acesso em 02 abr 2017]. Disponível: http:// dx.doi.org/10.1590/0034-7167.2015680626i.

29. Ruotsalainen JH,Verbeek JH,Mariné A, Serra C. Preventing occupational stress in healthcare workers. Cochrane Database Syst Rev.[Internet] 2015;(4)[acesso em 02 abr 2017].Disponível: http://dx.doi.org/10.1002/14651858. CD002892.pub5.

30. Coffeng JK,Boot CRL,Duijts SFA,Twisk JWR,Van Mechelen W,Hendriksen IJM. Effectiveness of a worksite social \& physical environment intervention on need for recovery, physical activity and relaxation; results of a randomized controlled trial. PLoS One.[Internet]2014;9(12) [acesso em 02 abr 2017]. Disponível: http://dx.doi. org/10.1371/journal.pone.0114860. 